\title{
RIEMANN'S METHOD AND THE PROBLEM OF CAUCHY
}

\author{
MONROE H. MARTIN
}

Introduction. The method devised by Riemann to solve the Problem of Cauchy applies to linear, hyperbolic, partial differential equations of second order for one unknown function $u$ of two independent variables $x, y$. For a homogeneous equation the essential points in the method are:

(a) The introduction of the characteristics as coördinate lines,

(b) The construction of a line integral $I=\int\{B d x-A d y\}$ which vanishes around closed paths, where:

(c) $A$ and $B$ are certain bilinear forms in $u, u_{x}, u_{y}$ and $v, v_{x}, v_{y}$, and,

(d) $v$ (Riemann's function) is a properly chosen two-parameter family of solutions of a second linear partial differential equation, the adjoint equation.

In this paper new bilinear forms are taken for $A, B$ in (b), and the rôle of the adjoint equation in (d) is taken over by a partial differential equation termed the associate equation of the original equation. Each solution $\phi$ of the original equation leads to an associate equation and the Problem of Cauchy is then solved with the aid of a properly chosen two-parameter family of solutions of the associate equation called the resolvent, the analogue of Riemann's function.

This modification offers some hope of extending Riemann's method to the Problem of Cauchy for linear hyperbolic partial differential equations with more than two independent variables. ${ }^{1}$ Such an extension to three independent variables is actually carried out in this paper for the equation of cylindrical waves. ${ }^{2}$ In the treatment of this equation, following (a), characteristic coördinates are adopted. As an interesting corollary, it turns out that axially symmetric solutions are governed by one ${ }^{3}$ of Euler's partial differential equations, namely,

An address delivered before the New Orleans meeting of the Society on April 20' 1951, by invitation of the Committee to Select Hour Speakers for Southeastern Sectional Meetings; received by the editors February 8, 1951.

${ }^{1}$ The classic work on Cauchy's problem is the book by J. Hadamard, Le problème de Cauchy et les équations aux dérivées partielles linéaires hyperbolique, Paris, 1932, where references to the work of other authors are given.

${ }^{2}$ My colleague A. Weinstein has kindly pointed out that an extension of Riemann's method for this equation has been given by H. Lewy, Verallgemeinerung der Riemannschen Methode auf mehr Dimensionen, Nachr. Ges. Wiss. Göttingen (1928) pp. 118-123. His method differs from ours in that it employs three "Riemann functions," and neither makes use of characteristic coördinates, nor of an "associate equation."

${ }^{3}$ See, for example, G. Darboux, Leçons sur la thêorie générale des surfaces, vol. 2, 2d ed., Paris, 1915, pp. 54-70. 


$$
(\alpha-\beta) u_{\alpha \beta}-\left(u_{\alpha}-u_{\beta}\right) / 2=0 .
$$

1. Riemann's method. The solution of the problem of Cauchy given by $\mathrm{Riemann}^{4}$ for the partial differential equation

$$
u_{x y}-a\left(u_{x}-u_{y}\right)=0, \quad a=a(x, y),
$$

has been extended ${ }^{5}$ to the general linear partial differential equation of second order in two independent variables $x, y$,

$$
u_{x y}+a u_{x}+b u_{y}+c u=f
$$

where $a, b, c, f$ are given functions of $x, y$.

In this section first we set forth the essentials of this method, as usually presented, to prepare the way for a modification which permits extension to an equation with three independent variables, the equation for cylindrical waves

$$
u_{x x}+u_{y y}-u_{t t}=0 .
$$

For simplicity we consider only equations of the type

$$
L(u)=u_{x y}-a u_{x}-b u_{y}=0, \quad a=a(x, y), \quad b=b(x, y) .
$$

Actually, if a solution $u_{1}$ of (2) and a solution $u_{0}$ of the homogeneous equation $(f \equiv 0)$ are known, equation (2) can always be reduced to one of type (4) for a new unknown function $\bar{u}$ defined by $u=u_{1}+u_{0} \bar{u}$.

The classical procedure associates with (4) its adjoint equation

$$
M(v)=v_{x y}+(a v)_{x}+(b v)_{y}=0,
$$

and is based ${ }^{6}$ on the differential identity

$$
\begin{gathered}
A_{x}+B_{y}=v L(u)-u M(v), \quad A=\left(v u_{y}-u v_{y}\right) / 2-a u v, \\
B=\left(v u_{x}-u v_{x}\right) / 2-b u v .
\end{gathered}
$$

This identity implies that the line integral

$$
I=\int\{B d x-A d y\}
$$

vanishes around closed paths lying in the interior of a domain $D$ within which the function $u, v$ are regular solutions of $L(u)=0$ and $M(v)=0$, respectively.

The fact that the line integral $I$ vanishes around closed paths leads immediately to the solution of Cauchy's problem for (4). Let $C$ be

\footnotetext{
${ }^{4}$ B. Riemann, Über die Fortpflanzung ebener Luftwellen von endlicher Schwingungsweite, Göttingen Abhandlungen vol. 8 (1858-1859) pp. 245-264, or Werke, Leipzig, 1892, 2d ed., pp. 156-175.

b J. Hadamard, op. cit. pp. 93-95.

J J. Hadamard, op. cit., pp. 81-82.
} 


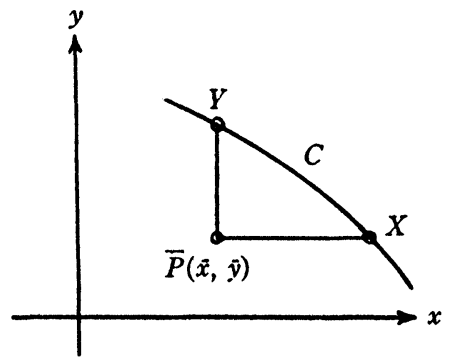

FIG. 1

an arc meeting any horizontal or vertical line in at most one point. Along $C$ the values of $u_{x}, u_{y}$ (Cauchy's data ${ }^{7}$ ) for the solution $u$ of (4) are given. In order to calculate the value $u(\bar{P})$ of the solution at the point $\bar{P}$ in the figure, the line integral $I$ is evaluated around the closed path $\bar{P} X Y \bar{P}$ formed by the horizontal line segment $\bar{P} X$, the $\operatorname{arc} X Y$ of $C$, and the vertical line segment $Y \bar{P}$. One finds

$$
\int_{\vec{P}}^{X} B(x, \bar{y}) d x+\int_{X}^{Y}\{B d x-A d y\}-\int_{Y}^{\bar{P}} A(\bar{x}, y) d y=0 .
$$

If a solution $v=v(x, y ; \bar{x}, \bar{y})$ of $M(v)=0$ can be found for which

$$
\begin{aligned}
& v_{x}=-b v \text { on } y=\bar{y}, \quad v_{y}=-a v \text { on } x=\bar{x}, \\
& v(\bar{x}, \bar{y} ; \bar{x}, \bar{y})=1
\end{aligned}
$$

hold on the characteristics $x=\bar{x}, y=\bar{y}$ through $\bar{P}$, we shall have

$$
A(\bar{x}, y)=(u v)_{y} / 2, \quad B(x, \bar{y})=(u v)_{x} / 2,
$$

and consequently (6) will yield

$$
u(\bar{P})=\frac{1}{2}[u(X) v(X)+u(Y) v(Y)]+\int_{X}^{Y}\{B d x-A d y\} .
$$

The function $v$ is known as Riemann's function, and, once it has been determined, $u(\bar{P})$ may be calculated by (9) from a knowledge of the Cauchy data along $C$.

The first step in Riemann's method is the formulation of the line integral $I$ so that it vanishes around closed paths. We note that the coefficients $A, B$ of the differentials are bilinear forms in the two sets of variables $u, u_{x}, u_{y}$ and $v, v_{x}, v_{y}$. Furthermore, the real reason for the

${ }^{7}$ In the usual terminology Cauchy data consist of the values $u_{0}$ of $u$ on $C$ and the values $u_{1}$ of its directional derivative in some direction not tangent to $C$. Assigning $u_{x}, u_{y}$ on $C$ determines $u_{1}$ exactly and $u_{0}$ up to an arbitrary additive constant. 
introduction of the adjoint equation $\left(4^{\prime}\right)$ and its solution $v$ is to make available the identity (5), which guarantees that $I$ vanishes around closed paths.

In seeking an alternative approach to the solution of Cauchy's problem we might take $A$ and $B$ to be general bilinear forms in $u, u_{x}, u_{y}$ and $v, v_{x}, v_{y}$ with coefficients arbitrary $y^{8}$ functions of $x, y$, and let the rôle of the adjoint equation be assigned to a partial differential equation,

$$
\Lambda(v)=v_{x y}-\alpha v_{x}-\beta v_{y}=0, \quad \alpha=\alpha(x, y), \beta=\beta(x, y),
$$

whose coefficients $\alpha, \beta$ are at our disposal.

With these general principles in mind, let us set $A=-\lambda^{-1} u_{y} v_{y}$, $B=\mu^{-1} u_{x} v_{x}$ and attempt to construct a line integral

$$
I=\int\left\{\lambda^{-1} u_{x} v_{x} d x+\mu^{-1} u_{y} v_{y} d y\right\}
$$

which vanishes around closed paths in the interior of a domain within which $u, v$ are regular solutions of $L(u)=0, \Lambda(v)=0$ respectively, the functions $\lambda, \mu$ of $x, y$ being, for the moment, perfectly arbitrary. ${ }^{8}$

From the identity

$$
\begin{aligned}
\left(\lambda^{-1} u_{x} v_{x}\right)_{y}-\left(\mu^{-1} u_{y} v_{y}\right)_{x}= & \lambda^{-1} \mu^{-1}\left[\left(\mu u_{x}-\lambda u_{y}\right) \Lambda(v)+\left(\mu v_{x}-\lambda v_{y}\right) L(u)\right] \\
& -\lambda^{-1} \mu^{-1}\left[\mu\left(\lambda^{-1} \lambda_{y}-a-\alpha\right) u_{x} v_{x}\right. \\
& +(a \lambda-\beta \mu) u_{x} v_{y}+(\alpha \lambda-b \mu) u_{y} v_{x} \\
& \left.-\lambda\left(\mu^{-1} \mu_{x}-b-\beta\right) u_{y} v_{y}\right],
\end{aligned}
$$

the analogue of (5), it is clear that $I$ will vanish around closed paths for every pair of solutions $u, v$ of $L(u)=0, \Lambda(v)=0$, provided the arbitrary functions $\alpha, \beta, \lambda, \mu$ satisfy

$$
\begin{array}{ll}
a \lambda-\beta \mu=0, & \lambda_{y}=(a+\alpha) \lambda, \\
\alpha \lambda-b \mu=0, & \mu_{x}=(b+\beta) \mu .
\end{array}
$$

When the equations in the first column are used to eliminate $\alpha, \beta$ from the equations in the second column, we find

$$
\lambda_{y}=a \lambda+b \mu, \quad \mu_{x}=a \lambda+b \mu .
$$

Consequently $\lambda_{y}=\mu_{x}$ and a potential function $\phi=\phi(x, y)$ exists for which

\footnotetext{
${ }^{8}$ Subject, of course, to the usual differentiability conditions.
} 


$$
\lambda=\phi_{x}, \quad \mu=\phi_{y},
$$

with $L(\phi)=0$.

Moreover the first column in (11) yields

$$
\alpha=\phi_{x}^{-1} \phi_{y} b, \quad \beta=\phi_{x} \phi_{y}^{-1} a \text {. }
$$

With this choice of $\alpha, \beta, \lambda, \mu$ the identity reduces to

$\left(\phi_{x}^{-1} u_{x} v_{x}\right)_{y}-\left(\phi_{y}^{-1} u_{y} v_{y}\right)_{x}=\left(\phi_{x}^{-1} u_{x}-\phi_{y}^{-1} u_{y}\right) \Lambda(v)+\left(\phi_{x}^{-1} v_{x}-\phi_{y}^{-1} v_{y}\right) L(u)$, where

$$
\Lambda(v)=v_{x y}-b \phi_{x}^{-1} \phi_{y} v_{x}-a \phi_{x} \phi_{y}^{-1} v_{y} .
$$

The equation $\Lambda(v)=0$ will be termed an associate equation of $L(u)=0$. Each solution $\phi$ of $L(u)=0$ gives rise to such an associate equation, of which it is automatically a solution.

LEMma. The line integral

$$
I=\int\left\{\phi_{x}^{-1} u_{x} v_{x} d x+\phi_{y}^{-1} u_{y} v_{y} d y\right\}
$$

vanishes around closed paths lying in a region $D$ within which $u$ is a regular solution of $L(u)=u_{x y}-a u_{x}-b u_{y}=0$ and $v$ is a regular solution of an associate equation $\Lambda(v)=v_{x y}-b \phi_{x}^{-1} \phi_{y} v_{x}-a \phi_{x} \phi_{y}^{-1} v_{y}=0$, the function $\phi$ being any solution of $L(u)=0$, regular in $D$, for which $\phi_{x} \neq 0$, $\phi_{y} \neq 0$.

Guided by the procedure in Riemann's method, we evaluate $I$ around the closed path $\bar{P} X Y \bar{P}$ in Fig. 1, obtaining, in place of (6),

$$
\begin{aligned}
\int_{\bar{P}}^{X} \phi_{x}^{-1} u_{x} v_{x} d x & +\int_{X}^{Y}\left\{\phi_{x}^{-1} u_{x} v_{x} d x+\phi_{y}^{-1} u_{y} v_{y} d y\right\} \\
& +\int_{Y}^{\bar{P}} \phi_{y}^{-1} u_{y} v_{y} d y=0 .
\end{aligned}
$$

Instead of the initial conditions (7) prescribed for $v$ on the characteristics through $\bar{P}$, we now require

$$
v_{x}=\phi_{x} \text { on } y=\bar{y}, \quad v_{y}=-\phi_{y} \text { on } x=\bar{x} .
$$

Once such a solution of the associate equation has been found, the integrands in the first and last integrals in (13) reduce to $u_{x},-u_{y}$ respectively, and one finds quite readily that

$$
u(\bar{P})=\frac{1}{2}[u(X)+u(Y)]+\frac{1}{2} \int_{X}^{Y}\left\{\phi_{x}^{-1} u_{x} v_{x} d x+\phi_{y}^{-1} u_{y} v_{y} d y\right\} .
$$


A solution $v=v(x, y ; \bar{x}, \bar{y})$ of an associate equation $\Lambda(v)=0$ subject to the initial conditions (14) is termed a resolvent of $L(u)=0$ and, after it has been found, $u(\bar{P})$ can be calculated by (15) from the Cauchy data for $u$ along $C$. Since each solution $\phi$ of $L(u)=0$ gives rise to an associate equation $\Lambda(v)=0$, there are, a priori, many such resolvent functions. In practice, one would, of course, choose for $\phi$ the simplest particular solution of $L(u)=0$ that is available.

THEOREM. The solution to Cauchy's problem for the partial differential equation $L(u)=u_{x y}-a u_{x}-b u_{y}=0$ with initial data prescribed along the arc $C$ in Fig. 1 is given by

$$
u(\bar{P})=\frac{1}{2}[u(X)+u(Y)]+\frac{1}{2} \int_{X}^{Y}\left\{\phi_{x}^{-1} u_{x} v_{x} d x+\phi_{y}^{-1} u_{y} v_{y} d y\right\},
$$

where $\phi=\phi(x, y)$ is any regular particular solution of $L(u)=0$ for which $\phi_{x} \neq 0, \phi_{y} \neq 0$, and $v=v(x, y ; \bar{x}, \bar{y})$, a resolvent of $L(u)=0$, is a two-parameter family of solutions of the associate equation

$$
\Lambda(v)=v_{x y}-b \phi_{x}^{-1} \phi_{y} v_{x}-a \phi_{x} \phi_{y}^{-1} v_{y}=0
$$

subject to the initial conditions $v_{x}=\phi_{x}$ on $y=\bar{y}, v_{y}=-\phi_{y}$ on $x=\bar{x}$, upon the characteristics through $P$.

Let us apply this theorem to equation (1) treated by Riemann. For the particular solution $\phi$ we select $\phi=x+y$, whereupon the associate equation becomes $\Lambda(v)=v_{x y}+a\left(v_{x}-v_{y}\right)=0$, the conjugate equation $^{9}$ to $L(u)=0$, and (15) reduces to

$$
u(\bar{P})=\frac{1}{2}[u(X)+u(Y)]+\frac{1}{2} \int_{X}^{X}\left\{u_{x} v_{x} d x+u_{y} v_{y} d y\right\} .
$$

When $a=m(x-y)^{-1}$ the equation $L(u)=0$ is Euler's equation, and explicit formulas for its resolvent have been given ${ }^{9}$ in terms of Appell's hypergeometric function $F_{1}$ of two variables.

2. The equation for cylindrical waves. Classical solution of Cauchy's problem. It has been known for a long time that the solution to Cauchy's problem

$$
u(x, y, 0)=u^{0}(x, y), \quad u_{t}(x, y, 0)=u^{1}(x, y),
$$

for the wave equation (3) is given by ${ }^{10}$

9 M. H. Martin, The rectilinear motion of a gas, Amer. J. Math. vol. 45 (1943) pp. 391-407.

${ }^{10} \mathrm{~J}$. Hadamard, op. cit. p. 71. 


$$
2 \pi u(\bar{x}, \bar{y}, \bar{t})=F_{\bar{t}}\left(u^{1}\right)+\frac{\partial}{\partial \bar{t}} F_{\bar{t}}\left(u^{0}\right),
$$

where the operator $F_{r}(f)$ operating on a function $f$ is defined by

$$
F_{r}(f)=\int_{0}^{2 \pi} \int_{0}^{r} \frac{f(\bar{x}+\rho \cos \phi, \bar{y}+\rho \sin \phi)}{\left(r^{2}-\rho^{2}\right)^{1 / 2}} \rho d \rho d \phi,
$$

or, on setting $\rho=r \tau$, by

$$
F_{r}(f)=r \int_{0}^{2 \pi} \int_{0}^{1} \frac{f(\bar{x}+\tau r \cos \phi, \bar{y}+\tau r \sin \phi)}{\left(1-\tau^{2}\right)^{1 / 2}} \tau d \tau d \phi .
$$

We shall give this classical solution another form so that it may more readily be compared with the solution to be obtained later by generalizing Riemann's method.

By differentiating (18) with respect to $r$ under the integration sign, we obtain

$$
\begin{aligned}
\frac{\partial F_{r}(f)}{\partial r}= & \frac{1}{r} \int_{0}^{2 \pi} \int_{0}^{r} \frac{f \rho}{\left(r^{2}-\rho^{2}\right)^{1 / 2}} d \rho d \phi \\
& +\frac{1}{r} \int_{0}^{2 \pi} \int_{0}^{r} \frac{\rho^{2} f_{\rho}}{\left(r^{2}-\rho^{2}\right)^{1 / 2}} d \rho d \phi .
\end{aligned}
$$

After an integration by parts in the first term, this reduces to

$$
\frac{\partial F_{r}(f)}{\partial r}=2 \pi f(\bar{x}, \bar{y})+\int_{0}^{2 \pi} \int_{0}^{r} \frac{r f_{\rho}}{\left(r^{2}-\rho^{2}\right)^{1 / 2}} d \rho d \phi,
$$

and with the necessary modifications in (17), (19), it is easy to verify that (16) takes the form

$$
u(\bar{x}, \bar{y}, \bar{t})=u^{0}(\bar{x}, \bar{y})+\frac{1}{2 \pi} \int_{0}^{2 \pi} \int_{0}^{\bar{t}} \frac{\bar{t} u_{\rho}^{0}+\rho u^{1}}{\left(\bar{t}^{2}-\rho^{2}\right)^{1 / 2}} d \rho d \phi .
$$

This is the desired form for the solution to Cauchy's problem. With either (16) or (20), the value $u(\bar{P})$ of the solution at the vertex $\bar{P}(\bar{x}, \bar{y}, \bar{t})$ of the characteristic cone $(x-\bar{x})^{2}+(y-\bar{y})^{2}=(t-\bar{t})^{2}$ is computed from Cauchy's data given over the circular region $(x-\bar{x})^{2}$ $+(y-\bar{y})^{2} \leqq \bar{t}^{2}$ intercepted by the characteristic cone on the carrier of Cauchy's data, the plane $t=0$. We note in passing that (20) may be given a concise form

$$
u(\bar{x}, \bar{y}, \bar{t})=u^{0}(\bar{x}, \bar{y})+\frac{1}{2 \pi} \int_{0}^{2 \pi} \int_{0}^{\bar{t}}\left(l_{\bar{t}} u_{\rho}^{0}-l_{\rho} u^{1}\right) d \rho d \phi
$$


by the introduction of the length $l=\left(\bar{t}^{2}-\rho^{2}\right)^{1 / 2}$ of the tangent line drawn from the vertex $\bar{P}(\bar{x}, \bar{y}, \bar{t})$ of the characteristic cone to a sphere of radius $\rho$ with center at the point $(\bar{x}, \bar{y}, 0)$ where the axis of the characteristic cone pierces the plane $t=0$.

3. The equation for cylindrical waves. Characteristic coördinates. Each point $\left(x_{0}, y_{0}, t_{0}\right)$ of $(x, y, t)$-space is a vertex for a characteristic cone $\left(x-x_{0}\right)^{2}+\left(y-y_{0}\right)^{2}=\left(t-t_{0}\right)^{2}$. For the computation of the value of the solution $u$ at a point $\bar{P}(\bar{x}, \bar{y}, \bar{t})$ we shall, for $\bar{t}>0$, be interested only in those points which lie on, or above, the plane $t=0$ and upon, or inside, the lower mantle of the characteristic cone with vertex at $\bar{P}$, that is, in the points of the conical region

$$
C: \quad 0 \leqq t \leqq \bar{t}, \quad(x-\bar{x})^{2}+(y-\bar{y})^{2} \leqq(t-\bar{t})^{2},
$$

pictured in Fig. 2a. In this figure $\bar{P} \bar{P}_{0}$ is the axis of the characteristic cone with vertex at $\bar{P}$. We introduce curvilinear coördinates $\alpha, \beta, \phi$

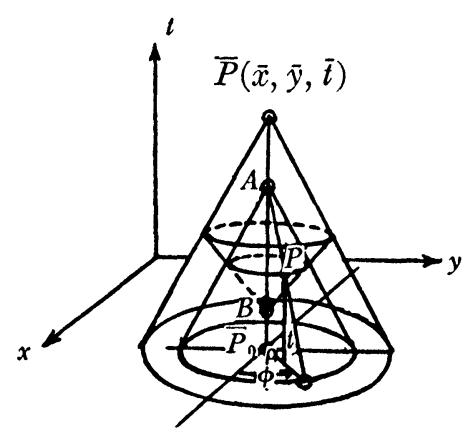

FIG. 2a. Conical region.

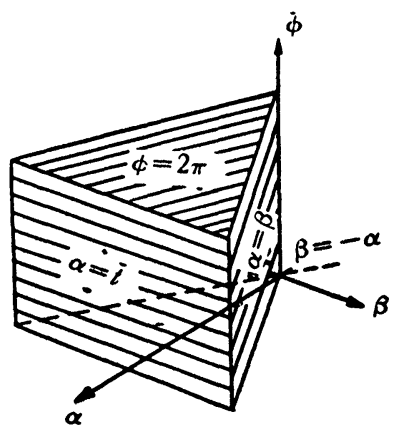

Fig. 2b. Wedge $W$.

to fix the position of a point $P(x, y, t)$ in $C$ by taking points $A(\bar{x}, \bar{y}, \alpha)$, $B(\bar{x}, \bar{y}, \beta)$ on the axis so that the lower mantle of the characteristic cone with vertex $A$ intersects the upper mantle of the characteristic cone with vertex $B$ in a circle through $P$. The position of $P$ on this circle is then fixed by the angle $\phi$ shown in Fig. 2a. Elementary geometrical considerations show that

$$
\begin{gathered}
x=\bar{x}+\frac{1}{2}(\alpha-\beta) \cos \phi, \quad y=\bar{y}+\frac{1}{2}(\alpha-\beta) \sin \phi, \\
\quad t=\frac{1}{2}(\alpha+\beta),
\end{gathered}
$$

and consequently, on writing $\rho=\left((x-\bar{x})^{2}+(y-\bar{y})^{2}\right)^{1 / 2}$, we shall have 


$$
t=(\alpha+\beta) / 2, \quad \rho=(\alpha-\beta) / 2 .
$$

The coördinates $\alpha, \beta, \phi$ of a point $P$ will be termed characteristic coördinates, even though only two of the coördinate surfaces through $P$ are mantles of characteristic cones, the third being a half-plane.

After the introduction of characteristic coördinates, equation (3) for cylindrical waves becomes

$$
L(u)=u_{\alpha \beta}-\frac{1 / 2}{\alpha-\beta}\left(u_{\alpha}-u_{\beta}\right)-\frac{u_{\phi \phi}}{(\alpha-\beta)^{2}}=0 .
$$

Obviously, we shall be interested only in those solutions of this equation which are periodic of period $2 \pi$ in $\phi$.

For solutions with axial symmetry about $x=\bar{x}, y=\bar{y}$ this equation reduces to Euler's equation

$$
u_{\alpha \beta}-\frac{m}{\alpha-\beta}\left(u_{\alpha}-u_{\beta}\right)=0, \quad m=1 / 2 .
$$

The transformation (21) carries the conical region $C$ of $(x, y, t)$ space into the wedge

$$
W: \quad 0 \leqq \alpha \leqq \bar{t}, \quad-\alpha \leqq \beta \leqq+\alpha, \quad 0 \leqq \phi \leqq 2 \pi,
$$

in $(\alpha, \beta, \phi)$-space shown in Fig. 2b. From (22) the base of $C$ becomes the vertical face $\beta=-\alpha$ in back of $W$; the conical mantle of $C$ transforms into the vertical face $\alpha=\bar{t}$ of $W$; while the axis $\bar{P} \bar{P}_{0}$ inside $C$, from (21), appears as the vertical face $\beta=\alpha$ of $W$. Both horizontal faces $\phi=0, \phi=2 \pi$ of $W$ are transforms of the trace of $C$ on the plane $y=\bar{y}$. The vertex $\bar{P}$ of $C$ transforms into the edge $\alpha=\beta=\bar{t}$ of $W$; the periphery of the base of $C$ becomes the edge $\alpha=\vec{t}, \beta=-\vec{t}$ of $W$; and the center $\bar{P}_{0}$ of the base of $C$ appears as the edge $\alpha=\beta=0$ of $W .^{11}$

\section{The equation for cylindrical waves. Solution of Cauchy's prob-} lem by Riemann's method. We begin by formulating Cauchy's problem in $(\alpha, \beta, \phi)$-space. The carrier $t=0$ for Cauchy's data in $(x, y, t)$ space becomes the plane $\alpha+\beta=0$ in $(\alpha, \beta, \phi)$-space. From (21) we find

$$
u_{\alpha}=\left(u_{\rho}+u_{t}\right) / 2, \quad u_{\beta}=-\left(u_{\rho}-u_{t}\right) / 2, \quad u_{\phi}=u_{\phi} .
$$

${ }^{11} \mathrm{I}$ am indebted to J. B. Diaz for drawing my attention to an intuitive process which carries the wedge $W$ into the cone $C$. One shrinks the face $\beta=\alpha$ of $W$ into the lower edge and brings the upper face of the resulting solid into coincidence with the lower face by revolution around this edge as axis. 
In particular, for the carrier $\alpha+\beta=0$, this yields

$$
u_{\alpha}=\left(u_{\rho}^{0}+u^{1}\right) / 2, \quad u_{\beta}=-\left(u_{\rho}^{0}-u^{1}\right) / 2, \quad u_{\phi}=u_{\phi}^{0},
$$

so that Cauchy's data $u_{\alpha}, u_{\beta}, u_{\phi}$ are prescribed on the carrier $\alpha+\beta=0$.

Thus the problem of computing the value $u(\bar{P})$ at the vertex $\bar{P}(\bar{x}, \bar{y}, \bar{t})$ of the characteristic cone in $(x, y, t)$-space, from a knowledge of Cauchy data given over the circular region intercepted by the characteristic cone on the plane $t=0$, becomes the problem of computing the value of a solution to (23) along the edge $\alpha=\beta=\bar{t}$ of $W$ when Cauchy data are prescribed on the face $\beta=-\alpha$ of $W$.

We have pointed out in $\$ 1$ that the first step in Riemann's method for two independent variables is the formulation of a properly chosen line integral $I$ which vanishes around closed paths. In seeking a generalization to partial differential equations with three independent variables, it is natural to search for a surface integral $I$ which vanishes when extended over closed surfaces.

For the partial differential equation (23) the proper surface integral is

$$
I=\iint\left\{u_{\beta} v_{\beta} d \beta d \phi-u_{\alpha} v_{\alpha} d \phi d \alpha+u_{\phi} \frac{v_{\alpha}-v_{\beta}}{(\alpha-\beta)^{2}} d \alpha d \beta\right\},
$$

where $u=u(\alpha, \beta, \phi)$ is a solution of $(23)$ and $v=v(\alpha, \beta)$ is a solution of the associate equation

$$
\Lambda(v)=v_{\alpha \beta}+\frac{1 / 2}{\alpha-\beta}\left(v_{\alpha}-v_{\beta}\right)=0,
$$

which, incidentally, is the conjugate equation to (24). That $I$ actually vanishes over closed surfaces is easy to verify, for if we set $K=u_{\beta} v_{\beta}$, $L=-u_{\alpha} v_{\alpha}, M=u_{\phi}\left(v_{\alpha}-v_{\beta}\right)(\alpha-\beta)^{-2}$, a computation shows that

$$
K_{\alpha}+L_{\beta}+M_{\phi}=0 \text {. }
$$

Keeping in mind the procedure in $\$ 1$, where we evaluated the line integral $I$ of Lemma 1 around the closed path $\bar{P} X Y \bar{P}$ in Fig. 1, we integrate $I$ in (26) over the surface of the wedge $W$. We obtain

$$
\underset{\alpha=\bar{t}}{I}+\underset{\beta=-\alpha}{I}+\underset{\beta=\alpha}{I}+\underset{\phi=0}{I}+\underset{\phi=2 \pi}{I}=0 .
$$

Since $u_{\phi}$ has period $2 \pi$ in $\phi$ and $v_{\alpha}, v_{\beta}$ are independent of $\phi$, the last two terms cancel each other, because the projection of the top face 
on the bottom face of $W$ has opposite orientation. When the integrations in the first three terms are written out explicitly, we obtain

$$
\begin{aligned}
\int_{0}^{2 \pi} \int_{-\bar{t}}^{+\bar{t}}\left[u_{\beta} v_{\beta}\right]_{\alpha=\bar{t}} d \beta d \phi & +\int_{0}^{2 \pi} \int_{0}^{\bar{t}}\left[u_{\alpha} v_{\alpha}-u_{\beta} v_{\beta}\right]_{\beta=-\alpha} d \alpha d \phi \\
& -\int_{0}^{2 \pi} \int_{0}^{\bar{t}}\left[u_{\alpha} v_{\alpha}+u_{\beta} v_{\beta}\right]_{\beta=\alpha} d \alpha d \phi=0 .
\end{aligned}
$$

The next step in the procedure for two independent variables was to impose conditions (14) on the characteristics through $\bar{P}$ for the solution $v$ of the associate equation (12). Corresponding to this, we require that the solution $v$ of the associate equation (27) meet the conditions

$$
v_{\beta}=1 \text { for } \alpha=\bar{t}, \quad v_{\alpha}=v_{\beta}=0 \text { for } \beta=\alpha,
$$

on the two faces of $W$ intersecting in the edge $\alpha=\beta=\bar{t}$ of $W$ representing $P$. The solution

$$
v=\alpha+\beta+2((\bar{t}-\alpha)(\bar{t}-\beta))^{1 / 2}
$$

of (27) actually meets these conditions, with

$$
v_{\alpha}=1-\left(\frac{\bar{t}+\alpha}{\bar{t}-\alpha}\right)^{1 / 2}, \quad v_{\beta}=1-\left(\frac{\bar{t}-\alpha}{\bar{t}+\alpha}\right)^{1 / 2} \text { for } \beta=-\alpha .
$$

When substitutions for $v_{\alpha}, v_{\beta}$ into (29) are made from (30), (31) we find that

$$
\begin{aligned}
2 \pi u(\bar{P})=\left.\int_{0}^{2 \pi} u\right|_{\alpha=\bar{t}, \beta=-\bar{t}} d \phi & \\
-\int_{0}^{2 \pi} \int_{0}^{\bar{t}}\left\{u_{\alpha}\right. & {\left[1-\left(\frac{\bar{t}+\alpha}{\bar{t}-\alpha}\right)^{1 / 2}\right] } \\
& \left.\quad-u_{\beta}\left[1-\left(\frac{\bar{t}-\alpha}{\bar{t}+\alpha}\right)^{1 / 2}\right]\right\}_{\beta=-\alpha} d \alpha d \phi,
\end{aligned}
$$

since $u=u(\bar{P})$ on the edge $\alpha=\beta=\bar{t}$ of $W$. This formula expresses the value $u(\bar{P})$ of the solution to (23) in terms of Cauchy data prescribed on the carrier plane $\beta=-\alpha$. The problem of Cauchy is solved in $(\alpha, \beta, \phi)$-space, and we shall now transform this solution back to $(x, y, t)$-space to obtain the classical result (20) as a check on our calculations. 
If, in addition to (25), we observe that $u=u^{0}(\bar{t}, \phi)$ on the edge of $\alpha=\bar{t}, \beta=-\bar{t}$ of $W$, formula (32) reduces to

$$
u(\bar{P})=u^{0}(\bar{x}, \bar{y})+\frac{1}{2 \pi} \int_{0}^{2 \pi} \int_{0}^{t} \frac{\bar{t} u_{\rho}^{0}+\rho u^{1}}{\left(\tilde{t}^{2}-\rho^{2}\right)^{1 / 2}} d \rho d \phi,
$$

that is, to (20), provided we note, from (22), that $\rho=\alpha$ when $\beta=-\alpha$.

UNIVERSITY OF MARYLAND 